\title{
Theorizing Glissant. Sites and citations, ed. John E. Drabinski and Marisa Parham
}

\section{Emanuela Cacchioli}

\section{(2) OpenEdition}

1 Journals

\section{Edizione digitale}

URL: http://journals.openedition.org/studifrancesi/5458

DOI: 10.4000/studifrancesi.5458

ISSN: 2421-5856

\section{Editore}

Rosenberg \& Sellier

\section{Edizione cartacea}

Data di pubblicazione: 1 dicembre 2016

Paginazione: 576-577

ISSN: 0039-2944

\section{Notizia bibliografica digitale}

Emanuela Cacchioli, «Theorizing Glissant. Sites and citations, ed. John E. Drabinski and Marisa Parham », Studi Francesi [Online], 180 (LX | III) | 2016, online dal 01 janvier 2017, consultato il 18 septembre 2020. URL : http://journals.openedition.org/studifrancesi/5458 ; DOI : https://doi.org/10.4000/ studifrancesi.5458

Questo documento è stato generato automaticamente il 18 settembre 2020.

\section{(c)}

Studi Francesi è distribuita con Licenza Creative Commons Attribuzione - Non commerciale - Non opere derivate 4.0 Internazionale. 


\title{
Theorizing Glissant. Sites and citations, ed. John E. Drabinski and Marisa Parham
}

\author{
Emanuela Cacchioli
}

\section{NOTIZIA}

Theorizing Glissant. Sites and citations, ed. by John E. DRABINSKI and Marisa PARHAM, London, Rowman\&Littlefield International, 2015, 175 pp.

1 Gli scritti teorici di Édouard Glissant rappresentano un punto di riferimento indiscusso per i critici che si occupano della cosiddetta letteratura francofona, in particolare per l'ambito caraibico e i cultural studies o più in generale per il postcoloniale. Il suo contributo monumentale spazia da riflessioni storiche e filosofiche a osservazioni estetiche e etnologiche. Il presente volume vuole essere un omaggio alla memoria di Glissant e, in particolare, alle risonanze filosofiche che pervadono la sua opera, anche in considerazione del fatto che è possibile rintracciare uno stretto legame tra l'autore della Martinica e alcuni concetti chiave della filosofia occidentale del Novecento. In primis, le nozioni di radice e di tradizione, da intendersi in ultima analisi come mezzi per sfuggire all'alienazione della vita quotidiana. Secondo i curatori del volume, Glissant elabora un modello di pensiero post-occidentale che rompe con i paradigmi tradizionali per giungere a una nuova visione dell'identità, in cui ogni elemento costitutivo è in relazione con gli altri. Lo scrittore parte da una frammentazione del radicamento dovuta alla cultura composita propria di ogni individuo dei Caraibi e converge sullintersezione tra trauma e bellezza. Il primo elemento corrisponde all'origine africana che Césaire e i teorici della Négritude avevano messo al centro della loro riflessione e delle loro rivendicazioni. Sebbene sia portatrice di dolore, la storia che caratterizza il centro America è anche ricca di momenti bellissimi che vale la pena ricordare e celebrare. Gli eventi e le principali figure del passato costituiscono, dunque, un centro di interesse e un motivo di creazione. Diviene un vero e proprio progetto 
critico (in quanto il passato è recuperato e reso produttivo) e poetico (il ripensamento della frammentazione permette di immaginare un mondo diverso). La visione profetica del passato è essenzialmente anticoloniale e rifiuta ogni forma di autorità per perseguire la molteplicità. Esemplificative a tal proposito le metafore della mangrovia $\mathrm{e}$ della radice. Questi, oltre alle forme che assume l'Antillanité e le risonanze filosofiche implicite e esplicite, sono i temi centrali dei contributi critici riuniti nel presente volume. Nel primo saggio H. Adlai MURDoch, Glissant's "Opacité" and the ReConceptualization of Identity (pp. 7-27), si focalizza sull'opacità intesa come elemento indispensabile della resistenza e della creatività dell'autore. Tale aspetto permette inoltre una ridefinizione della soggettività e dell'identità intese come una relazione dinamica. Seanna Sumalee OAKLEY, In Citation to the Chance: Glissant, Citation, Intention, and Interpretation (pp. 29-52), ricostruisce la presenza del pensiero filosofico di Bergson nelle opere di Glissant ed in particolare nei testi poetici. Oltre a rintracciare l'influenza che il primo ha avuto sul secondo, è possibile comprendere meglio il complesso legame che l'autore della Martinica stabilisce con la pratica poetica surrealista. Sono Gilles Deleuze e Félix Guattari i teorici convocati nel contributo di Clevis HEADLEY, Glissant's Existential Ontology of Difference (pp. 53-83). Il critico si pone l'obiettivo di descrivere l'ontologia di Glissant in relazione alla materialità della storia. In merito a questo aspetto, l'autore non si presenta come un mero imitatore delle teorie di Deleuze e Guattari, bensì come un innovatore che ha introdotto concetti rivoluzionari per rendere conto della complessa realtà caraibica. Max HANTEL, Toward a Sexual Difference Theory of Creolization (pp. 85-102), sceglie la teoria della differenza sessuale elaborata da Luce Irigaray per analizzare e approfondire il concetto di creolizzazione. Il critico indaga le modalità attraverso le quali quest'ultima modifica le idee. Il testo di Hanetha vÉTÉ-CONGOLO, "The Ripening"'s Epic Realism and the Martinican Tragic Unfulfilled Political Emancipation (pp. 103-127), offre un'attenta lettura del romanzo La Lézarde al fine di mettere in luce la posizione politica di Glissant e la sua resistenza di fronte alla scelta di trasformare la Martinica in Dipartimento. Come il titolo del contributo suggerisce, Breadfruit, Time and Again: Glissant Reads Faulkner in the World Relation (pp. 129-138), Marisa PARHAM rintraccia nelle opere dello scrittore la presenza di un'intertestualità che ha come oggetto la riflessione sulla storia elaborata da William Faulkner. John E. DRABINSKI, Aesthetics and the Abyss: Between Césaire and Lamming (pp. 139-164), conduce un'analisi simile alla precedente e giunge alla conclusione che l'idea di sradicamento e di deterritorializzazione estetica siano un modo per oltrepassare i limiti che il senso di perdita di Césaire e l'umanesismo esistenziale di Lamming avevano fissato nelle loro teorie. La collettanea si chiude con un breve testo poetico di Neil ROBERTS che vuole essere un omaggio a Glissant. Il titolo del componimento è: Marronage between Past and Future: Requiem for Edouard Glissant (pp. 165-166).

2 Che si tratti degli scritti teorici, dei romanzi o delle pagine dedicate alla poesia, l'opera di Glissant non cessa di parlare ai critici e di suggerire loro nuove prospettive di indagine come è avvenuto per questa raccolta di saggi. Il Glissant filosofo che viene presentato in Theorizing Glissant sottolinea ancora una volta, se mai ce ne fosse bisogno, la grande caratura dell'autore e la sua influenza in ambito letterario, estetico e critico. Il volume suggerisce, inoltre, che ogni sua opera è permeata da una riflessione filosofica molto articolata e complessa in grado di coniugare la sua visione originale con quella dei più grandi pensatori del Novecento. 